\title{
SOBRE LA HIPERMETRÍA EN LOS SEGUNDOS "GOZOS" DE LA VIRGEN, DEL LIBRO DE JUAN RUIZ
}

Voy a ocuparme en el presente estudio del problema métrico que presentan los versos 39ade, 40ab, 41abd y 42ae del Libro de Buen Amor (LBA). Diez versos eneasilábicos incrustados extrañamente en una sextilla simétrica $a a b: a a b$ (octosílabos los $a$, pentasílabos los $b$ ), en vez de los esperados octosilábicos, interpretados como regulares por un importante sector de la crítica editorial ${ }^{1}$ de la obra de Juan Ruiz (JR). Dedicaré también atención a los versos de pie quebrado de estos "Gozos de Santa María". Federico Hanssen $^{2}$ es el primero que aplica a la lírica castellana (concretamente, a

1 Utilizo las siguientes abreviaciones y siglas para referirme a los editores del $L B A$ y a algún otro notable crítico del mismo: Sz (T. A. Sánchez: Madrid, 1790), Jnr (F. Janer: Madrid, 1864; reimpr., 1966), Duc J. Ducamin: Toulouse, 1901), Cej (J. Cejador: Madrid, 1914; $11 .^{\text {a }}$ ed., 1967-70), Ld (M. R. Lida : Buenos Aires, 1941; reimpr., 1973), Chi (G. Chiarini: Milán-Nápoles, 1964), CrN (M. Criado de Val y E. W. Naylor: Madrid, 1964; 2. a ed., 1972), Bol (A. Bolaño: México, 1967; 6. ${ }^{\mathrm{a}}$ ed., 1980), Cor (J. Corominas: Madrid. 1967), Rfl (J. Rafael Fontanals y L. Pons: Barcelona, 1971; 7. a ed., 1982), Ws (R. S. Willis : Princeton, 1972), Jt (J. Joset: Madrid, 1974 ; reimpr., 1981), RRiv C. Real de la Riva: Madrid, 1975), Zs (A. N. Zahareas y S. R. Daly: University Park, 1978), Jaur (P. Jauralde: Tarragona, 1981), Blc (A. Blecua : Barcelona, 1983), Hn (F. Hanssen: $A U C h, 1902$; reimpr., 1958), Ag (J. M. Aguado: Madrid, 1929) y MM (M. Morreale: $R F E$, 1983). Me refiero a los manuscritos del $L B A$ según es usual: $S$ (ms. de Salamanca), $G$ (ms. Gayoso) y $T$ (ms. de Toledo), con expresión de versos ( $26 \mathrm{~d} S, 498 \mathrm{c} G S, 1139 \mathrm{~b} T)$ o folios (G34v, S63r, T14v). En el cuerpo del artículo transcribo y sigo el texto del $L B A$ establecido por el Profesor Jacques Joset.

2 F. Hanssen, "Los metros de los Cantares de Juan Ruiz", AUCh, 110-11 (1902), 
la del Arcipreste de Hita) el artificio trovadoresco advertido y descrito por Adolfo Mussafia unos años antes, como propio de la poesía provenzal y galaico-portuguesa. Tal artificio (al parecer, aceptable a la percepción y sensibilidad rítmico-poética del hombre medieval) consiste, según Mussafia y Hanssen, en el uso permisible de versos con sílaba final aguda hipermétrica, respecto a los normales de la estrofa de modo que, en la escansión de los mismos, la dicha sílaba final cuenta, a los efectos, como si fuera de sólo un tiempo (y no de dos, como por posición le correspondería), con lo cual se iguala así ficticia o artificialmente, en los versos en cuestión, la medida métrica con la silábica o, lo que es lo mismo, la medida métrica de estos versos con la de los demás correspondientes de la estrofa ${ }^{3}$. No me he detenido en analizar con qué extensión se da tal principio métrico en la poesía provenzal y galaico-portuguesa. En la medieval castellana es, en verdad, infrecuente, si se excluye lo que parece darse en el $L B A$ ("leçión e muestra de metrificar e rimar e de trobar", Pról., líns. 152-53) particularmente en algunas de las poesías de corte más provenzal, p. ej., en la cantiga a la Virgen de 1406a$1048 \mathrm{~g}$ y en su subsiguiente "Pasión de Cristo", 1049a-1058g 4 . Ciertamente, en estas páginas no intento negar a la poesía de Juan Ruiz un recurso métrico admitido sin excepción desde Hanssen ${ }^{5}$ por todos aquellos críticos que han abordado el problema métrico descrito ( $P$. Henríquez Ureña, T. Navarro Tomás, Cor, Ws, Jaur, MM, etc.). Pero sí quisiera cuestionar la generalización, aparentemente un tanto desorbitada, a que se ha llevado tal catalexia en la restauración textual del $L B A$. Qué duda cabe que la aceptación en los cantares ruicianos de un principio así resuelve sin complicaciones mayores la medida de una larga cincuentena de versos masculinos hipermétricos del tipo en cuestión. Ahora bien, los manuscritos del $L B A$ dan cumplida muestra del estado lamentable en que se encuentran muchos de sus versos. Un cotejo de aquéllos arroja por doquier constantes deformaciones, alteraciones, sustituciones o eliminaciones de partículas y palabras. Luego, hay que concluir que, al igual que en tantos más casos de versos de manifiesta

págs. 161-220. Citaré por su reimpresión en los Estudios. Métrica. Gramática. Historia literaria, del Profesor Hanssen (Santiago de Chile, 1958, vol. III, págs. 167-223; ad hoc, págs. 190, 198, 208, etc.

3 Por ejemplo, en el $L B A$ 1048cd "yo en tu memoria / algo quiero fazer", el verso $d$ de seis sílabas, pero de siete tiempos métricos por ser masculino, se conforma con la medida poética del hexasílabo $c$, en función del recurso descrito.

- Se citan en el $L B A$ unos sesenta versos del tipo, los que podrían reducirse a una docena escasa, si se tiene en cuenta los probables errores de transmisión en muchos de ellos y una distinta concepción estrófica para 33a-43f y 1049a-1058g. Véase al respecto, D. C. Clark, Morphology of Fifteenth Century Castilian Verse (Pittsburgh-Lovaina, 1964), pág. 62 ; $L B A$ Cor, págs. $405 \mathrm{~b}-406 \mathrm{a} ; L B A \mathrm{Jt}$, ad 1046 y sigs., etc.

5 "Esta particularidad que se encuentra en algunas poesías portuguesas la ha aclarado el señor Mussafia, i por esto la llamo la lei de Mussafia", F. HAnssen, "Los metros ...", pág. 208. 
deficiencia métrica debida a corrupción textual de copia, al menos algunos de los de la dicha cincuentena (justificados cómodamente con la llamada ley de Mussafia) deben presentar exceso de medida, no en razón del artificio provenzal, sino como resultado de una torpe o descuidada alteración de alguna de las palabras que los constituyen y que, por lo tanto, requieren más adecuadamente la aplicación del tradicional principio de la emendatio, efectuada, por supuesto, con la moderación y prudencia exigidas por la Filología Clásica en su riguroso sistema crítico-editorial. Más concretamente ya, en los sesenta y seis versos de arte menor constitutivos de las once sextillas simétricas de estos "Gozos de Santa María" la collatio de los materiales de la tradición diplomática existentes da un saldo de nada menos que 51 variantes entre $S$ y $G$ (laguna en $T$ ), lo cual equivale a una correspondencia por verso de 0.77 . Indice de errores particularmente significativo, habida cuenta de que estos versos son de corte breve (los 48 octosílabos, con una medida de cinco palabras por verso, y los 22 pentasílabos, de tres por verso). Con base, pues, en tal fundamento de polo negativo, pasemos ya a analizar la estructura de los versos ruicianos dichos:

\section{El quinto fue de grand dulçor $39 a$}

La lección "de grand dulçor" la fijan así todos los editores de esta poesía de JR, salvo en detalles intrascendentes a nuestro objeto ( $\mathrm{Sz}, \mathrm{Jnr}$, Jaur; $\mathrm{Hn}^{\circ}$, Cej y Bol, "de grant dolçor"; Cor, Rfl y Ws, "de grand dolçor"; RRiv, Zs; Blc, MM), sin duda, porque registrándose así en los códices ("de grand dulçor" $S$, "de grant dolçor" $G$ ), la ley de Mussafia da base para justificar el exceso de metría del verso. Pero, la medida octosilábica del mismo se haría cabal, sin distorsionar el sentido de la frase, suprimiendo la partícula "de", supuestamente adicionada al sintagma por un copista de apógrafo previo y común a $S G$ : "El quinto fue grand dulçor" ?.

\section{al çielo, a su Padre Mayor 39d}

Los editores, siguiendo al ms. $S$, establecen el verso $39 \mathrm{~d}$ como lo refleja la forma de Jt, aquí transcrita (Ws opta por $G$ : “a. c. al su P. mayor"). A mi ver, el "su" de $S G$ es, con toda probabilidad, partícula hipocorística interpolada por un copista temprano, quizás llevado inadvertidamente por el posesivo paralelo "tu" de 49b. Sin tal partícula la metría del verso queda

- F. Hanssen, "Los metros ...", pág. 201.

7 El comentado es un tipo de error muy frecuente en el $L B A$, semejante a los de a "Dios en ti enbía" $24 \mathrm{~d} S$, por "Dios enbía" $G$; "cerca del pulgar" 55bS, por "çerca el pulgar" $G$, etc. La enmienda que sugiero me parece preferible a la supresión del verbo, propuesta por Ag ("el quinto de grand dulçor", Glosario, pág. 115). 
ajustada a la de ocho tiempos requeridos, sin que precise el recurso descrito por Mussafia: "al çielo, al Padre mayor". Restituyo en el verso el artículo contracto presente en $G$. La propuesta supresión es, creo, enmienda conservadora y de base legítima, ya que la adición (o supresión) del adjetivo posesivo es error muy común en los códices del $L B A^{8}$.

\section{e tú finqueste con amor $39 \mathrm{e}$}

Hn, Chi, Cor, Jt, Rfl, Ws y MM establecen este verso de JR como aquí se transcribe; Ag y los editores Sz, Jnr, Cej, Bol, Jaur, Zs y Blc difieren de aquéllos en presentar la variante "fincaste" del ms. $S$, la que es también de mi preferencia " (Sz y Jnr, además, fijan “et" en vez de “e”). Es decir, todos los editores respetan con máximo rigor conservador la hipermetría del verso por constar así en $S G$, optando por justificar implícita o explícitamente tal exceso de medida en función de la ley de Mussafia. Un criterio editorial moderadamente menos conservador, pero (creo) no falto de validez por su lógica argumentativa, permitiría también aqui ajustar tal verso cataléctico al isosilabismo métrico correspondiente y propio del mismo en una sextilla simétrica mediante la aplicación de la emendatio. El verso lo permite en dos maneras posibles, ambas sin estridencias:

1. a Eliminando del verso, por espúreo, el pronombre enfático "tú". Es enmienda obvia y la más natural de las soluciones que se proponen. El "tú", aunque (por supuesto) inexistente en el texto original de JR, está implícitamente presente en el sufijo desinencial de la forma de perfecto "fincaste/finqueste" $S G$. Nada más fácil para un torpe escriba, que copia con escasa atención un apógrafo del poema, arquetipo más o menos remoto de $S G$, que el exteriorizar inadvertidamente este tú inmerso y oscuro en la referida desinencia (-aste/-este), dando así realce emocional, con inconsciencia, al sujeto de la oración transcrita o, lo que es lo mismo, a la sustancia poética de 39ef. La interpolación de los pronombres-sujeto (como es la del caso) es error muy corriente en los manuscritos medievales, en particular, la de los monosilábicos (yo, tú, él, nos, vos). El mismo LBA ofrece ejemplos sobreabundantes de ello, algunos muy paralelos al del caso que nos

8 Por ejemplo: "por sus signos" 59bG, en lugar de "por señas" $S$, probablemente; "dexa me de tus roydos" 742aS, por "dexadme de roído" ("dexat me de rroydo" $G)$, etc. Ag propuso "facial su Padre mayor" : facial en vez de "al çielo" de $S$ o "al çielo, al" de $G$, es enmienda violenta e infundada y, por lo tanto, desechable.

- La preferencia para el $L B A$ de las formas de perfecto en -aste, frente a las de sus alternancias en -este, las discuto en mi artículo "Problemas de crítica textual en el Libro de Buen Amor: vv. 1a-10d y Prólogo", en vías de publicación en Thesaurus. 
ocupa: "si quieres tú ${ }^{10}$ amor" $430 \mathrm{aG}$, frente a "si quisieres amar" $S$ (regularizo las grafías paleográficas en estos y los subsiguientes ejemplos por no ser transcendentes); "los fazes tú ${ }^{11}$ ençender" $1565 \mathrm{c} T$, en vez de "los fazes ençender" $S$; "mas yo le mando" $366 \mathrm{c} G$, frente a "pero mando" $12 S$; "quantas yo vi" $706 \mathrm{a} S$, en vez de "quantas nunca vi" $G$; "e yo só perdido" $794 \mathrm{~d} S$, en vez de "e só perdido" $G$; "fue yo probar" $950 \mathrm{~b} G$, frente a "fui a provar" $S T$; "do quier que él sea" $1314 \mathrm{a} T$, frente a "do quier que sea" $S G$; "el martes él quisiese ir" $1372 \mathrm{c} G$, frente a "el martes quisiese ir" $S T$; “yo sería escarnida" $1481 \mathrm{~d} T$, frente a "sería mal escarnida" $S G$, etc. ${ }^{13}$.

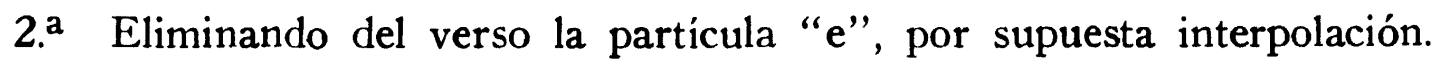
Enmienda asimismo posible, aunque (a mi ver) de menos visos de probabilidad. El escriba de un apógrafo previo, pero éste de tronco estemmático todavía común a $S G$, pudo haber incorporado tal " $\mathrm{e}$ " inadvertidamente al comienzo de 39e para suavizar el brusco corte de lo que en el poema ruiciano, en su forma original, debiera haber sido (en esta segunda hipótesis) dos cláusulas asindéticas e independientes. Lo que, en tal supuesto, constituiría el "gozo quinto" de la Virgen, sería únicamente, en la estrofa, su visión de la ascensión de Jesús al cielo ${ }^{14}$ (39bcd); la oración subsiguiente ("tú fincaste con amor / de a êl ir" 39ef) sería concebida, no ya como parte integrante del dicho "gozo", sino como simple reflexión del poeta sobre la

10 Los editores del $L B A$ establecen el v. 430a, unos conforme a $S(\mathrm{Sz}, \mathrm{Jnr}, \mathrm{Cej}$, Bol, Chi, Zs, Jaur, Blc) y otros siguiendo a $G$ (Cor, Jt, Rfl, Ws); pero todos ellos, salvo Rfl, sin incorporar la lección "tú" de $G$ al texto crítico establecido, evidentemente, por haber considerado espúrea tal partícula. Valga decir, no obstante, que la métrica no rechaza per se la inclusión de este pronombre en el verso, según aparece en el ms Gayoso. De ahí que $\mathrm{Rfl}$, con buena lógica, lo haya recogido en su edición.

11 El metro, ciertamente, permitiría la inclusión de "tú" en el verso; pero lo más probable, como piensan todos los editores del $L B A$, es que el dicho personal sea error incorporado, en vista de los precedentes "tu" 1565 y y "tú" $1565 \mathrm{~b}$, colocados también en segundos hemistiquios.

12 Al menos, todos los editores fijan el verso sin el "yo". Obviamente, por considerarlo lección interpolada ("pero mando", Sz, Jnr, Zs, Jaur; "mas le mando", Cej, Bol, Cor, Rfl, Ws; "mas mando", Chi, Jt, Blc). Ahora bien, la combinación de las lecturas de $G$ y $S$, "mas yo mando", sería también aceptable y, aun, a mi parecer, con buen grado de probabilidad, ya que la explicitud del pronombre en este caso concreto refuerza y realza considerablemente la orden emitida a base de la presencia en la oración del sujeto que la manda ("yo": 'el juez').

$18 \mathrm{Ni}$ qué decir tiene que el error de omisión del "tú" (al igual que el de otros pronombres personales en funciones de sujeto) es también muy corriente en los viejos manuscritos; p. ej., en el $L B A$ : "[tú] nunca paras mientes" 373aS; "quando [tú] non coidares" $579 \mathrm{~d} G$; "qual [tú] demandares" $1002 \mathrm{~b} G$ (en $S$, "qual [tú] demandudieres"), etc.

14 Nótese que esto es también sólo lo que Juan Ruiz expresa como la quinta de las "alegrías" de la Virgen en su tercera y cuarta redacción de los "Gozos": "Quando a los çielos sobió, / quinto plazer tomaste" 1640ab (Jt, "tomeste"); "quinto, do Jesús es / al çielo sobido, / e lo vido" 1645 efg (Jt, "Jhesús"). 
consecuencia de tal visión. Entre ambas cláusulas, pues, así interpretadas, se precisaría un punto y como o dos puntos ${ }^{15}$. He aquí cómo queda la estrofa con las enmiendas sugeridas, según las dos posibilidades expuestas para el v. 39e:

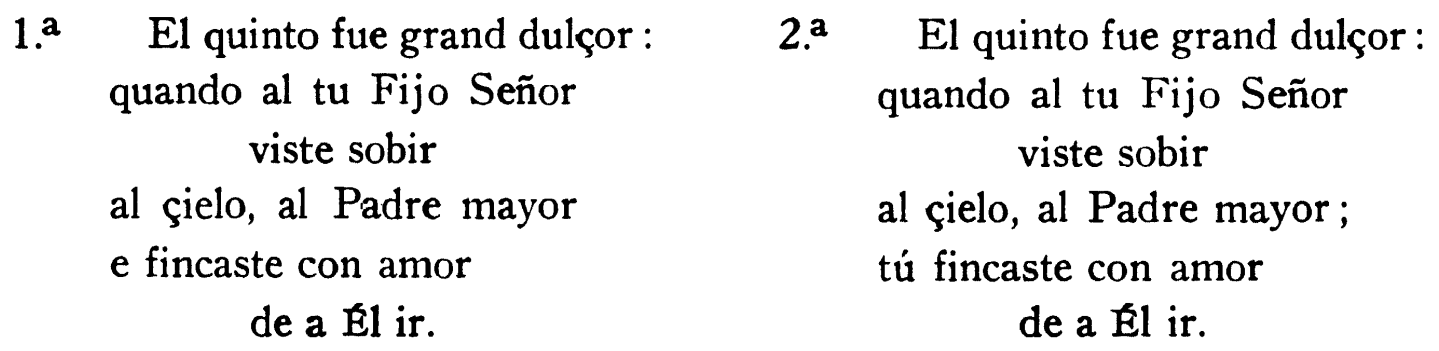

Non es el sesto de olvidar $40 \mathrm{a}$

En los manuscritos del $L B A$ se presenta el verso como sigue: "Este sesto non es de dubdar" $S 3 \mathrm{r}$; "non es el sesto de oluidar" G2v. Como se ve, Jt, seguido por Rfl, Ws y Blc, fija el verso en su edición sin alterar en nada la lectura existente en $G$. Por lo que se deduce que, para la aceptación métrica del mismo (eneasílabo masculino, o terminado en voz oxítona) el editor belga ( $y$ sus seguidores en este punto) con toda probabilidad se basa en el recurso de Mussafia. Pero, parece más natural el rectificar la forma adverbial "non" a "no", como hacen otros editores, siguiendo la enmienda sugerida por Ag (Glosario, pág. 115): "no es el sesto de olvidar”, Cej, Bol, Chi, Cor y MM. Tal rectificación es mínima: en los manuscritos la $n$ final del adverbio se suele indicar con el signo típico de abreviación, según costumbre general de época; si en el original ruiciano no constaba en este concreto lugar, es muy fácil que algún copista posterior lo inscribiera inadvertidamente, siguiendo la fuerza de la costumbre. Ahora bien, en tal reconstrucción me parece ser preferible la lección "dubdar" de $S$ a la de "olvidar" de $G$, aceptada generalmente por casi todos los editores desde 1914: creo más probable la sustitución de "dubdar" por "olvidar", por ser este verbo de uso común más popular, que la de "olvidar" por "dubdar". Zs, más ajustado al texto de $S$, presenta "non" sin corregir, y "dubdar".

Los códices del $L B A$ dan lugar a una segunda restauración del verso, a mi juicio, aún quizás más oportuna que la propuesta por $\mathrm{Ag}$, porque se acopla mejor a la estructura del verso presente en $S$. A saber: enmienda de "non" SG a "no", según queda dicho; pero manteniendo el adverbio en la

15 Por lo demás, la adición de la partícula " $\mathrm{e}$ " es también error muy común en los manuscritos del $L B A$, p. ej.: "e yo, desque vi" $106 a S$; "e dixo" $969 \mathrm{c} G$; "e dixe le yo" $997 \mathrm{~g} G$; "e mucho toçino" $1373 \mathrm{~b} G$; "e si a mí" $1389 \mathrm{a} G$; "e faré por vos" $1651 \mathrm{bG}$, etc. 
posición interna que consta en $S$, y preferencia de "el" $G$ al "este" de $S$, aunque respetando el orden del ms. $S$. El v. 40a, con tal restauración quedaría ya con la medida justa, así: "El sesto no es de dubdar" ${ }^{16}$.

\section{los disçiplos ${ }^{17}$ vino alunbrar $40 \mathrm{~b}$}

Sz, Jnr, Cej, Bol, Rfl, Zs, Jaur y Blc presentan el verso con nueve sílabas o tiempos métricos, tal y como consta en el ms. $S$ : "los disçipulos vino alunbrar"; Cor, Jt, Ws y MM, siguiendo a $\mathrm{Hn}$, reproducen este mismo texto en sus respectivas ediciones (MM, art. cit., pág. 226); pero con la grafía vulgar "diciplos" (Jt, "disçiplos" "disciplos", diferencia intranscendente) en vez de "disçípulos", con lo cual el verso se acorta en una sílaba, permitiendo interpretarlo ya como métricamente válido (es decir, como octosilábico), sobre la base de que el artificio trovadoresco descrito por Mussafia fue, en efecto, aplicado también aquí intencionalmente por JR. Nosotros, con $\mathrm{Ag}$, preferimos fundamentar este v. $40 \mathrm{~b}$ en la lectura del $\mathrm{ms}$. $G$, aplicándola dos ligeras intervenciones: una rectificación a su grafía "alubrar", lección obviamente equivocada, y una enmienda menor a su forma "diçipulos": "los disçiplos alunbrar". Con tal arreglo el verso adquiere la medida octosilábica exigida por su número de orden en la sextilla simétrica, dejando el contexto del "sexto gozo" con pleno sentido para la mente del cristiano, a la que intencionalmente va dirigida esta piadosa poesía.

$\mathrm{El}$ reparo más serio que presenta esta reconstrucción es el de la validez de "disçiplos" (Ag, "diciplos"), como forma original de JR, pues en los códices del $L B A$ no existe caso alguno explícito de la forma diş̧iplo, $-\boldsymbol{s}^{18}$.

$18 \mathrm{Sz}$ y Jnr se limitaron a transcribir el verso siguiendo a $S$; pero no pusieron esfuerzo alguno para arreglar el problema métrico que tal estructura implica. Y Hn, para solucionar éste, introdujo una enmienda violenta ("Est sesto non es de dubdar"), con razón, no aceptada por los sucesivos editores de estos "Gozos de Santa María".

17 Corrijo el "disciplos" del $L B A \mathrm{Jt}$, por suponerlo errata de edición. Jt refleja sistemáticamente la asibilación de la $c$, cuando corresponde, con la ce sedilla (c).

18 En $S G$ constan nueve casos de tal sustantivo (ninguno en $T$ ). Cinco de los cuales con valor relativamente útil para el problema planteado, pues en ellos la $u$ interna de disçipulo, -s se representa con el característico signo inscrito medieval de la abreviación: $30 \mathrm{~b}, S 3 \mathrm{v} ; 40 \mathrm{~b}, S 4 \mathrm{v} ; 427 \mathrm{a}, S 29 \mathrm{r} ; 1049 \mathrm{~g}$, G51r y 1646b, $S 99 \mathrm{v}$. En los restantes casos la sílaba - pu- aparece bien definida e incuestionable: "diçipulos" 30a, G2r; "diçipulos" 40b, G2v; "disçipulo" 427a, G15r; "disçipulo" $1049 \mathrm{~g}$, S62r. Incidentalmente, $\mathrm{CrN}$ dicen que la grafía paleográfica de G1049g puede leerse "diçiplo" (página 644b); así leyó también tal locus Cor (pág. 415, ap. crit.), lo cual no se comprende, porque el rasgo típico de la abreviación de la $-u$ - en el manuscrito $G$ corre inscrito, clara y distintamente, desde el comienzo de la $s$ hasta la $o$, atravesando el palo de la $l$. Signo que hace imposible obviamente la lectura indicada por $\mathrm{CrN}$ y Cor. Un ligerísimo punto que presenta la $l$ en su parte alta es, por supuesto, intranscendente. Añadiré que la misma incorrección expresa Jt en su aparato crítico: "G: diçiplo (posiblemente diçipulo)", $L B A \mathrm{Jt}$, II, pág. 72, y que Chi hace otro tipo de error: "disçipulo' SG" 
Ahora bien, siendo tal forma un vulgarismo medieval corriente y, por otra parte, exigiendo la métrica del verso $40 \mathrm{~b}$ tal síncopa, creemos justificada la introducción de la dicha enmienda en el $L B A$. Al fin y al cabo, es reducción mínima de las formas constantes en sus códices existentes. Por supuesto, varios editores y críticos del $L B A$ aceptan en sus respectivos estudios tal reducción vulgar, como enmienda oportuna, en este y otros loci del poema ${ }^{19}$.

tú estavas en ese lugar, 40d

En GS se registra el v. como aquí se transcribe. Así que no les sobra razón a los editores del $L B A$ para fijarlo en sus respectivos textos con tal estructura, en el entendimiento implícito (es de suponer) de que acuden a la llamada ley de Mussafia para justificar su excesiva medida métrica. Sin embargo, el proponer aquí, como enmienda, la sustitución del demostrativo "ese" por el artículo "el" no parece implicar un grave quebranto al respeto debido a la tradición manuscrita del $L B A$. Razones: $1 .^{\mathbf{a}}$, porque, salvo el matiz deíctico de "ese", ambas partículas poseen un valor morfosintáctico próximo, o muy semejante, y un mínimo contenido lógico de apoyo; por lo que es comprensible que los escribas suelan fácilmente intercambiarlas al copiar con cierta desatención los textos viejos. 2. ${ }^{\text {a }}$, porque los manuscritos existentes del Libro de JR dejan sobreabundante evidencia de que este tipo de error de transmisión de texto (el intercambio de demostrativos $\mathrm{y}$ artículos) aparece reflejado en otros muchos loci de la obra ${ }^{20}$. Y $3 .^{2}$, porque la enmienda propuesta permite rectificar, con mínima violencia al texto dado, el (creo) irregular anisosilabismo del v. 40d, aportando así solución lógica (supongo) al problema discutido ${ }^{21}$.

Este seteno non ha par: $41 \mathrm{a}$

(pág. 201, ap. crít., ad v. 4323). Chi además fija el v. 40b como está en $G$ y así, con exceso de medida.

10 Véase al respecto $30 \mathrm{~b}(\mathrm{Hn}, \mathrm{Cor}, \mathrm{Jt}$, Ws y MM), 40b $(\mathrm{Hn}$, Cor, Jt, Ws y Jaur), 1040g (Hn, 1049h; Chi, 1049d); Cor (1049h; Jt y Ws, 1049h) y 1646d (Hn, Cor, Jt Ws y MM).

20 Por ejemplo: "Este sesto non" 40aS, frente a "no es el sesto" $G$ (precisamente en la misma estrofa del verso aquí discutido); "este seteno" $41 \mathrm{a} G$, frente a "el septeno" $S$ (que de inmediato comentamos); "esa ora fue" $542 \mathrm{~d} S$, frente a "a la ora fue" $G$; "conortad me esta llaga" $605 \mathrm{c} S$, frente a "e conortat la llaga" $G$; "buhona destas que venden" $669 \mathrm{a} S$, frente a "buhona delas que venden" $G$; "çerca de esa mata" $952 \mathrm{~b} G$, frente a "çerca de una mata" $S T$; "ando por esta sierra" $998 \mathrm{~b} S$, frente a "ando la syerra" $G$; "en çima de ese puerto" $1006 \mathrm{c} G$, frente a "ençima del puerto" $S$; “abras / ese blaço (¿'brazo'?)" $1031 \mathrm{c} S$, frente a "abras / el moço" G; "por esa claustra", $1307 \mathrm{~b} T$, "por esa calastra" $G$, frente a "por la caustra" $S$, etc.

$21 \mathrm{Ag}$ (Glosario, pág. 115) sugirió el cambio de "estavas" por "eras". Enmienda radical y extrema que, si arregla la metría del v. 40d, es infundada. 
Hanssen $(A U C h, 1902)$ fue el primero que fijó tal verso con la variante "este", tomada de $G$, a pesar de que ella hace al mismo eneasílabo. Le siguen en tal decisión Cor, Rfl, Ws, Blc y MM, sin duda, porque en función de la ley de Mussafia, estos editores interpretan el dicho verso como de medida octosilábica y, por tanto, válido. La lección "el" del ms. $S$ ("El septeno non ha par") tiene la ventaja de eliminar una hipermetría cuestionable y su aceptación en el establecimiento del verso queda dentro del margen permisible a la crítica textual más rigurosa. Es ésta la lección incorporada al $L B A$ tradicional desde $\mathrm{Sz}$ : Jnr, Cej, Bol, Chi, Zs, Jaur y Blc, editores con los que nosotros también concurrimos. $Y$, ya sin mayor insistencia, en el supuesto de que la lección original de JR fuera "este" y no "el", cabría todavía un arreglo legítimo del verso para evitar el tener que aplicarle el recurso de Mussafia: simplemente, el hacer caso omiso del signo representativo de la $n$ final del adverbio "non" de los manuscritos, por la razón expuesta al discutir el v. 40a. Insistiré algo más en ella. Los códices del $L B A$ presentan el adverbio sistemáticamente con el signo característico de abreviación de la $n$ final ${ }^{22}$, lo que, dado lo avanzado de la diacronía del castellano, no implica que tal signo paleográfico equivalga siempre a la expresión implícita de la tal $-n$. En muchos casos la inscripción de aquél es, sin duda, simple hábito anodino de escritura o resultado de desatención al copiar ${ }^{23}$. Es decir, para los copistas del $L B A$, carentes del rigor crítico exigido al scholar actual, la grafía paleográfica en cuestión muchas veces significa, en efecto, "non"; pero otras tiene que significar necesariamente "no". Por ser éste punto importante en la crítica textual del $L B A$ aduciré aquí algunos argumentos de base:

1. ${ }^{\circ}$ Ante todo, la existencia en los manuscritos de formas reducidas "no" explícitas (muy escasas, en verdad) asegura la concurrencia en el

22 Salvo error de cuenta, advierto en $S 790$ formas de "non" ó el $91.5 \%$ del total de las formas del adverbio de negación presentes en el códice, frente a $48(5.6 \%)$ "non" y 25 (2.9\%) "no", de las cuales últimas, 20 son contracciones "nol" y 2 contracciones "nos"; en G, $835(99.1 \%)$ formas "non", frente a $5(0.6 \%)$ "non" y $2(0.3 \%)$ "no", una de las cuales es contracta "nol"; y en $T, 274$ (98.2\%) "non", ninguna forma "non" y $5(1.8 \%)$ "no", de las cuales 3 son contracciones "nol".

28 La reducción no es relativamente temprana, aunque durante mucho tiempo sigue concurriendo con la forma plena (non), sin duda, más en unas regiones o comarcas del dominio lingülstico castellano que en otras. De aquí que ni siquiera en los manuscritos del siglo XII (o principios del XIII) pueda asegurarse cuándo la grafía del adverbio con la inscrita abreviación significa efectivamente "non" y cuándo "no". Sobre la edición paleográfica del Poema de Mio Cid de R. Mentendez Pidal (3. ${ }^{2}$ ed. corregida: Madrid, 1956) he contado 133 (32\%) "non", 192 (46.2\%) "non" y 90 (21.7\%) "no", de los cuales últimos 29 son contractos "nol" ('no le') y 17 contractos "nos" ('no se'). Nuestro Diccionario de Referencias del "Poema de Mio Cid" (Ottawa: Carleton University Press, 1982) se basa en el texto crítico de Menendez Pidal, de aquí que no sea útil para tal recuento. 
$L B A$ de ese tipo de formas con el de las plenas ${ }^{24} .2 .^{\circ} \mathrm{La}$ existencia en los manuscritos de las formas contractas "nol" ('no le') y "nos" ('no se') refuerza la seguridad de la antedicha concurrencia en el $L B A^{25}$. 3. ${ }^{\circ}$ La profusión de la forma reducida no, presente en muchos de los códices de la segunda mitad del siglo xiII y del siglo xiv, es, a mi ver, claro indicio de que en los manuscritos del $L B A$ la discutida grafía adverbial abreviada ("non") no siempre representa non ni en la mente del escriba ni en la realidad. Así, por ejemplo, en los manuscritos de las dos Partes de la Estoria de España (mss. Esc. Y.I.2 y Esc. X.I.4), trabajos de los scriptoria regalia de Alfonso X y Sancho IV a finales del siglo xirI y principios del siguiente y en los que intervienen no menos de cinco copistas, constan, en la Parte I, 1305 (55.9\%) casos de "no", incluídos 51 de "nol", 10 de "noles", 1 de "nolo" y 3 de "nolos", frente a $833(35.6 \%)$ de "non" y $197(9.4 \%)$ de "non", y en la Parte II, 87 (2.8\%) casos de "no", frente a $2.034(64 \%)$ de "non" y 1.054 (33.2\%) de "non" ${ }^{26}$. En el códice de la Parte I de la General Estoria (B.N. de Madrid, ms. 816) hallo, contando por las Concordances de Kasten y Asociados, 211 (3.8\%) casos de "no" (de los que 191 son contractos con pronombre), frente a $849(14.6 \%)$ de "non" y $4747(81.6 \%)$ de "non", y en la Parte $I V$ de la misma Gen. Est. (Bibl. Vaticana, ms. Urb. Lat. 539), aparecen, según las antedichas Concordances, 229 (4.6\%) casos de "no" (incluídos contractos), frente a 4.145 (84\%) de "non" ${ }^{27}$ y $562(11.4 \%)$ de "non". En el apógrafo existente del Libro de Apolonio (ms. Esc. K.III.4), transcrito hacia 1370, constan 41 (10.9\%) casos de "no" (14 de los cuales, contractos), frente a $177(47 \%)$ de "non" y 158 (42\%) de "non" 28. En la

\footnotetext{
24 Véase 869dS ("non" en $G$ ), 1011bS ("non" $G$ ), 1395aS ("non" $G T$ ), 1518d $T$ ("non" GS), $1120 \mathrm{c} T$ ("non" GS) y 467cG ("non" $S$ ).

25 Véase para "nol": 101d $S, 187 \mathrm{~d} S, 212 \mathrm{~d} S, 227 \mathrm{c} S, 238 \mathrm{~d} S, 256 \mathrm{a} S, 359 \mathrm{~b} S$ ("non le" $G T$ ), 558cG, 636d $S, 641 \mathrm{a} S, 719 \mathrm{c} S$ ("non le" $G$ ), 904d $S$ ("non le" $G T$ ), 1033e $S$ ("non le" $G$ ), 1090cS ("non se" $G$ ), 1208d $S$ ("non le" $T$; "non se" $G$ ), 1219a $S T$ ("non" le" G), 1274c $S C, 1280 \mathrm{~d} S$ ("non le" GT), 1359d $S T$ ("non G), 1380aS ("non" $G T$ ), 1479cSG ("non" T), 1479dST ("non le" G), 1558aST, 1630bT ("non" $S$ ) y $1630 \mathrm{c} T$ ("non le" $S$ ); y para "nos": 662c $G$ (dos casos), 977a $S$ ("se" $G$ ) y 1032d $S$ ("non se" $G$ ). Desestimo en el cómputo la contracción "nom" ('no/non me') de 562aG y los raros casos de la misma contenidos en algunos de los manuscritos, que voy a citar en texto, por no ser posible determinar cuál de las dos formas del adverbio es la que corresponde al primer componente.
}

2 Hago el cómputo por las Concordances and Texts of the Royal Scriptorium Mamuscripts of Alfonso X, El Sabio (Madison, 1987) de L. Kasten, J. Nittii y Asociados. La discrepancia de casos explícitos de "no" en las dos Partes se explica aquí también por la antedicha insignificancia formal del signo de abreviación sobre la negación para el escriba del tiempo.

27 No deja de sorprender el alto porcentaje de casos explícitos de "non" en este manuscrito. Quizá ello sea debido a regionalismo del escriba, a hábito suyo de escritura o, incluso, a norma "editorial".

28 Libro de Apolonio, ed. E. Alarcos Llorach (Madrid, 1976), 3 vols. Hago el cómputo por el texto paleográfico dispuestos por el editor. 
Biblia romanceada $8.1 .4^{29}$, cuyo manuscrito se copia también a mediados del siglo XIV, aparecen nada menos que 1.430 (37.6\%) casos de "no" (72 de ellos, formas contractas), frente a 906 (23.7\%) de "non" y 1.469 (38.6\%) de "non". 4. " Los repetidos casos de variantes alternantes "no"/ "non", "nol" /"non le" y "nos"/"non se" presentes en estos manuscritos del LBA (así como en otros medievales), según se deja ver en los puntos $1 .^{\circ} \mathrm{y} 2 .^{\circ}$, reflejan bien el escaso valor que tenía el signo de abreviación referido para los copistas (y escritores) de los siglos XiI y y x y que éste se escribía sobre el "no" por simple costumbre e indiferencia, sin que representara ya, en muchos casos, la $n$ final, fonéticamente ya perdida o en vías avanzadas de perderse ${ }^{30}$. 5. ${ }^{\circ} \mathrm{Y}$, finalmente, la medida de muchos versos del Arcipreste exige el interpretar necesariamente ciertos casos de "non" constantes en los códices del $L B A$ como formas reducidas, p. ej., además del caso que nos ocupa, los 43a, 153d, 710c, 717d, 729a, 863a, 885d, 926g, 992g 1020d, $1222 \mathrm{~d}$ y $1294 \mathrm{~d}$. Y de hecho, varios editores del poema admiten en el texto por ellos dispuesto algunos de los casos de "no", en vez de "non", aquí señalados ${ }^{31} \mathrm{y}$ aun otros no resaltados por nosotros. El caso más extremo al respecto es el de M. R. Lida, quien llega a generalizar en su edición escolar la forma reducida del adverbio; Corominas presenta también esta forma con frecuencia, guiado siempre por necesidades métricas, según criterio propio, y lo mismo hacen los profesores Joset y Rafel Fontanels, aunque éstos más moderadamente que aquél; A Bolaño, que también suele alternar algo el "no" con el "non" (particularmente en la segunda mitad de la obra), parece no guiarle principio de metría poética alguno en ello.

\section{quando por ti quiso enbiar $41 b$}

Dos intervenciones criticas ha tenido este verso hasta ahora en la historia editorial del $L B A: 1^{\text {a }}$, la reflejada aquí por Jt ("enbiar"), también presentada por Cor ("embiar"), Chi y Blc, que arranca de Hn. Con ella, al interpretarse el verbo como trisílabo, el verso resulta hipermétrico, por lo que se deduce que tales editores suponen que JR aplicó aquí una vez más el artificio que Mussafia comentó. 2. a, la sustitución de "quando" ( $S G)$ por "do"; enmienda reciente de MM, por suponer que este adverbio "do", de

29 Edición de M. G. Littlefield (Madison, 1983). Sigo las concordancias en microfilm de esta obra.

so Evidentemente (y como consecuencia de lo antedicho), el hombre medieval, en su lectura de poesías, tenía que hacer caso omiso de los signos de abreviación expletivos sobre "no", cuando la métrica del verso exigía una sinalefa entre el "non" y la sílaba abierta inicial de la palabra subsiguiente, así como en otros casos. Hay que decir lo mismo respecto a la partícula "nin".

81 43a: Cor y Jt; 153c: Cor; 729a: Cor y Rfl; 863a: Rfl; 962g: Cor, Jt y Blc; 992g: Bol, Cor, Ws, Jt y Blc; 1222d: Cor y Rfl, y 1294d: Cor, Rfl y Jt. 
uso peculiar o arcáico, fue desechado por los copistas del $L B A$ en favor del más común "quando" no sólo en este pasaje, sino también en otros donde el ámbito temporal y espacial es próximo e intranscendente ${ }^{\mathbf{3 2}}$. La idea, sugerida ya en 1929 por J. M. Aguado para el "quando" de 1640g, fue aprovechada en dificultades textuales semejantes por Cor $(41 \mathrm{~b}, 1621 \mathrm{~b}, 1621 \mathrm{c}$, $1640 c, 1645 e)$, por Jt (25b, 41b, 1640c, 1645e), por Blc (41b) y ahora, ya más profusamente (pero, como simple "hipótesis de trabajo") por MM (25b, 29d, 30b, 35b, 41b, 1640c, 1645e). Como éste es punto importante que afecta gravemente a la rectitud textual de muchos pasajes del $L B A$, voy a detenerme brevemente en él (haciendo un paréntesis a nuestro objeto principal) para exponer las razones de base de mi rechazo de tal enmienda.

1. El remoto vínculo lingüístico indoeuropeo que unía a las partículas cuando $(<$ quando) y $d o(<d e-u b i)$ en su etimología y morfología quedó diluido mucho antes de la época clásica latina. Y, por supuesto, en la Edad Media castellana éstas están totalmente diferenciadas, semántica y morfológicamente, por lo que parece impropio filológicamente el atribuir equivalencia a ambas partículas. Claro que una hipotética sustitución de "quando" por "do" en ciertos textos (p. ej., en 68b, ó en 69a, ó en 689d, ó en 690a, etcétera) no alteraría radicalmente el sentido fundamental de la frase; porque tales partículas implican meras circunstancias de valor secundario y, a los efectos (los del conllevar la idea general, o propósito final, del autor) tan intranscendente y tan inoportuna (u oportuna) es la expresión del ámbito temporal (quando), como la del espacial $(d o)$. Ahora bien, en cuanto a tales matices circunstanciales se refiere, al sustituir cualquier "quando" por un "do", o viceversa, el significado de la oración queda sustancialmente modificado en ese respecto secundario. El hombre medieval, sin duda, diferenciaba los conceptos de ambas partículas tan distintamente como el de la actualidad ${ }^{\mathbf{3}}$.

2. Nueve son los casos de "quando" enmendados a "do" por unos u otros editores del $L B A$. Y siete de estos "quando" constan así en $G S$, los

32 El Profesor Corominas, que también aplica esta enmienda en otros loci del Libro de JR, según indico inmediatamente, expresa, como justificación de ello, la misma idea, pero de forma más taxativa (cf. $L B A$ Cor, pág. 604ab, ad $1645 \mathrm{c}$; véase también otra razón -a mi ver, petitionis principium; pero, en todo caso, ya de menor fuerza argumentativa - en pág. 598a, ad 1621bc). La realidad es que do es corriente en toda época en poesía, por lo que difícilmente podía habérseles hecho partícula extraña o arcaica a los copistas del $L B A$. En 'éste consta, por lo menos, unas noventa y nueve veces. Indice de frecuencia de los más altos existentes en la obra de Juan Ruiz.

33 Por curiosidad, vengo poniendo atención descle hace algún tiempo, en los aparatos críticos de las ediciones de las obras medievales consultadas, a las posibles variantes quando/do presentes en los mismos. El único caso que he hallado lo comento inmediatamente en la nota 37. 
manuscritos que aportan el pasaje correspondiente : 25b, 29b, 30b, 41b, 1621b, 1621c. Resulta difícil el comprender que en tales loci, ya $G$ ya $S$ no presenten ni una sola variante "do", supuestamente original de JR por aquellos editores, si es que, en efecto, el "do" hubiera sido sustituido por los copistas (por considerarlo ya arcáico) con el "quando", base en que se fundan los antedichos editores para justificar tal enmienda.

3. Dada la hipótesis de que los copistas de los apógrafos del $L B A$ alteran algunos "do" originales al más común "quando" por razones de arcaísmo, resulta todavía más inconcebible que en los setenta y siete loci de la obra de JR, donde la lección "quando" es coincidente en dos o en los tres códices ${ }^{34}$, no haya ni un solo caso de variación "quando"/ "do" entre ellos. Hay, sí, siete casos de otro tipo de variantes ${ }^{35}$.

4. Y, paralelamente, resulta, asimismo, extremadamente incomprensible en el mismo supuesto el que, de los setenta y dos loci con "do" coincidente en $S G$ o en $S T$ o en $S G T$ ", el $L B A$ sólo muestra una variante "quando" frente a "do" $(454 \mathrm{c} S / G){ }^{37}$, además de otra de distinta naturaleza $\mathrm{y}$, por tanto, ajena al problema que nos ocupa ("do"/"o" $29 \mathrm{~d} G / S$ ).

34 En $25 \mathrm{~b} S G, 26 \mathrm{~b} S G, 28 \mathrm{~b} S G, 29 \mathrm{~b} S G, 30 \mathrm{~b} S G, 35 \mathrm{bd} S G, 36 \mathrm{~b} S G, 37 \mathrm{~b} S G, 38 \mathrm{~b} S G$, 39a $S G, 41 \mathrm{~b} S G, 46 \mathrm{~d} S G, 95 \mathrm{a} S G, 97 \mathrm{ad} S G, 132 \mathrm{~b} S G, 356 \mathrm{a} S G, 401 \mathrm{~b} S G, 406 \mathrm{ac} S G, 407 \mathrm{c} S G$, $455 \mathrm{a} S G, 469 \mathrm{c} S G, 505 \mathrm{c} S G, 507 \mathrm{~b} S G, 522 \mathrm{a} S G, 524 \mathrm{c} S G, 564 \mathrm{a} S G, 579 \mathrm{~d} S G, 603 \mathrm{~b} S G, 614 \mathrm{~b} S G$, 695bSG, 745bSG, 799bSG, 807bSG, 809d $S G, 833 \mathrm{~d} S G, 865 \mathrm{c} S G, 867 \mathrm{~d} S G, 869 \mathrm{~d} S G, 870 \mathrm{~b} S G$ $871 \mathrm{~b} S G, 883 \mathrm{c} S G, 884 \mathrm{ab} S G, 887 \mathrm{~b} S G, 893 \mathrm{~b} S G, 902 \mathrm{a} S G, 957 \mathrm{a} S G, 999 \mathrm{c} S G, 1001 \mathrm{c} S G$, $1090 \mathrm{~d} S G, 1159 \mathrm{~b} S G, 1167 \mathrm{~d} S G, 1203 \mathrm{~b} S G, 1356 \mathrm{~b} S G T, 1357 \mathrm{~b} S G T, 1360 \mathrm{c} S G T, 1361 \mathrm{~d} S G T$, 1365bSGT, 1382bSGT, 1408a $S G T, 1411 \mathrm{a} S G T, 1459 \mathrm{a} S T, 1530 \mathrm{~b} S G T, 1532 \mathrm{~b} S G T, 1534 \mathrm{a} S G T$, $1536 c S G T, 1559 \mathrm{a} S T, 1621 \mathrm{~b} S T, 1621 \mathrm{dc} S T, 1652 \mathrm{c} S G, 1653 \mathrm{a} S G$, además de los casosvariantes a los que me refiero en la nota subsiguiente.

35 "Quando" / "elque" 356G/S (error en $G$ ), "quando" / "si" 357bS/G (probable lección original en $G$ ), "quando" / "quanto" $412 \mathrm{c} G / S$ (error en $G$ ), quando" / "desque" 577 a $G / S$ (dudoso, aunque creo más probable ser lección original el "quando" de $G$, en vista del "desque" de $576 \mathrm{~b}$, que quizá atrae con error a la variante segunda), "quando" / "quanto" $901 \mathrm{c} G / S$ (error en $S$ ) y "quando" / "quanto" 1138a $S G / T$ (error en $T$ ).

36 En 26d $S G, 29 \mathrm{~d} G / S, 63 \mathrm{~d} S G, 68 \mathrm{~b} S G, 69 \mathrm{a} S G, 330 \mathrm{c} S G, 365 \mathrm{~d} S G, 375 \mathrm{a} S G T, 391 \mathrm{~b} S G$, 394d $S G, 397 \mathrm{~d} S G, 404 \mathrm{c} S G, 418 \mathrm{c} S G, 425 \mathrm{c} S G, 473 \mathrm{~d} S G, 492 \mathrm{~d} S G, 493 \mathrm{a} S G, 498 \mathrm{a} S G, 499 \mathrm{~d} S G$, $506 \mathrm{~b} S G, 508 \mathrm{~d} S G, 513 \mathrm{~d} S G, 515 \mathrm{~d} S G, 523 \mathrm{~d} S G, 547 \mathrm{a} S G, 567 \mathrm{~b} S G, 599 \mathrm{c} S G, 721 \mathrm{~d} S G, 722 \mathrm{~b} S G$, $889 \mathrm{~b} S G, 993 \mathrm{~b} S G, 997 \mathrm{a} S G, 998 \mathrm{~b} S G, 1012 \mathrm{~d} S G, 1040 \mathrm{~b} S G, 1041 \mathrm{e} S G, 1078 \mathrm{a} S G, 1108 \mathrm{~b} S G$, 1137abc $S T G, 1139 \mathrm{~d} S G T, 1151 \mathrm{abc} S G T, 1156 \mathrm{c} S G, 1157 \mathrm{a} S G, 1174 \mathrm{~b} S G, 1176 \mathrm{c} S G, 1198 \mathrm{~b} S G$, $1198 \mathrm{c} G T / S$ ( $S$, "donde"), 1249bSGT, 1282c $S G T, 1284 \mathrm{c} S G T, 1298 \mathrm{~d} S G T, 1303 \mathrm{c} S G T$, 1308bSGT, 1309d $S G T, 1312 \mathrm{~b} S G, 1314 \mathrm{a} S G T, 1339 \mathrm{~b} S G T$ ( $T$, "dan" : 'do han'), 1243bSGT, $1365 \mathrm{c} S G T, 1376 \mathrm{a} S G T, 1377 \mathrm{c} S G T, 1381 \mathrm{~d} S G T, 1405 \mathrm{~d} S G T, 1484 \mathrm{~d} S G T, 1547 \mathrm{~d} S T, 1564 \mathrm{~b} S T$, $1569 \mathrm{c} S T, 1574 \mathrm{~b} S T$ y $1627 \mathrm{a} S T$.

37 Y, en este caso, hay que notar que la sustitución del "do" (en mi criterio, así como en el de Chi, Cor, Rfl, Ws, Jt y Blc, el original de JR) al "quando" no la hace el copista de $G$ (o cualquiera que sea el responsable de este error en la rama estemmática $G)$ por considerar ya arcaico aquel adverbio, sino por mera distracción, llevado 
Los indicados 148 casos de lecciones "quando" y "do" coincidentes en $S G, S T$ o $S G T$, frente a una sola divergencia "do" " "quando" en $454 \mathrm{c} G / S$ (y ésta justificada como error de carácter distinto al aquí discutido, véase nota 37) arrojan un índice de porcentaje cumplidamente elevado favorable a las coincidencias (99.3\%, frente a $0.7 \%)$. Sólido fundamento, creo, para desestimar como inoportunas las citadas nueve enmiendas "do" introducidas en el $L B A$ por los antedichos editores y críticos. En cualquiera de estos discutidos nueve casos de "quando" es preferible, a mi ver, la apócope "quand" como corrección (si oportuna) a la de "do" ${ }^{38}$. Pues con tal rectificación la lección manuscrita recibida, además de no perder un ápice de su imagen acústica y significado, sufre mínima distorsión formal. Bien es verdad que "quand" es arcaísmo inexistente en los códices conocidos del $L B A$, pero no es menos cierto que es forma todavía viva en el siglo xIII $\mathrm{y}$, sin duda, también en el siglo xiv, al menos, consta en muchos de los manuscritos copiados a lo largo de este siglo ${ }^{39}$. Lo cual implica que "quand", en modo alguno, era forma extraña a JR y que el poeta-arcipreste podía haber hecho uso de ella a conveniencia métrica.

Centrando ya la atención de nuevo en el v. $41 \mathrm{~b}$ del $L B A$, bien puede aplicarse al mismo la enmienda "quand" en vez de "quando" $(S G)$, caso de que quiera entenderse el verso transmitido como eneasílabo Con ello se daría una solución suave y no perturbadora a la hipermetría. Pero, a mi juicio, ni siquiera esto es necesario, ya que el verso es regular en medida si "enbiar" se lee - como creo debe hacerse - como forma bisílaba (en-biar). Esto es, diptongando sus dos vocales últimas ( $i a)$. Es licencia poética legítima que permite conservar íntegramente el texto de JR, en este caso, común a los dos manuscritos que lo aportan. Conviene tener presente, además, que en la zona norte de La Alcarria, limítrofe a la provincia de Zaragoza y donde JR pasa su vida, al menos en la actualidad, es relativamente frecuen-

por la sonoridad, en su subconsciente, del "quando" inmediato (454b) en frase acabada de copiar y similar a la que se copia: "non ayas miedo d'ella quando tienpo tovicres, // vergüença non te enbargue do $(S)$ / quando $(G)$ con ella estodieres".

s8 $\mathrm{Ni}$ qué decir tiene que "quand" es enmienda ya admitida por varios editores (Chi, Cor, Jt) en diversos loci del $L B A$.

39 Aparece, en efecto, en el códice de los Milagros de Berceo (R. A. E., ms. $A^{\prime} . A^{\prime \prime}$ ), copiado hacia 1330: vv. 138a, 447a; en el manuscrito del $L$. de Apolonio, copiado hacia 1370 : vv. 30a, 66d, 129a, 131c, 337d, 399c, 504b, 557c, 566c y 578c; en el códice escurialense de la citada Biblia romanceada, copiado a mediados del siglo xiv : fols. 95 r, 100r, 102r (dos veces) y 105r; en los manuscritos del L. de Alexandre (el de Osuna -B. N. de Madrid, ms. $V-5$, n. 10- copiado a fines del s. xiII o a principios del siguiente; el de París - B. N. de París, ms. esp. 488- copiado a principios del s. xv): vv. $485 \mathrm{~d} O, 604 \mathrm{~d} O, 1014 \mathrm{c} P, 1563 \mathrm{~d} P, 2180 \mathrm{~d} O$, etc., citados por $\mathrm{D}$. A. Nelson en su edición de esta obra (Madrid, 1978), pág. 81; lugar en donde se aporta también interesantes estadisticas de quand frente a quando. 
te tal tipo de diptongación vulgar en muchas voces de estructura paralela a enbiar por la fuerte influencia del uso de entonación navarro-aragonés.

\section{al çielo te fizo pujar $41 d$}

La hipermetría real de este verso la justifican los editores implícita o explícitamente por el recurso de Mussafia. Nosotros la suponemos ser consecuencia de un error originado en un apógrafo perdido del $L B A$ común a $S G$. Una enmienda (a mi entender, natural y lógica) que rectificaría la medida octosilábica, sin presuponer el referido recurso, es el cambio del orden de palabras en el verso: "te fizo al çielo pujar" ${ }^{40}$. No cabe duda que tal tipo de alteración (aceptado con extrema demasía en alguna de las ediciones del $L B A$ ) es modificación mayor que exige siempre una firme base de apoyo para ser recibido como críticamente aceptable. En el discutido caso parece justificarlo la métrica misma requerida por el verso en cuestión, circunstancia reforzada por la estructura de la frase, cuyo comienzo sugiere tener trazas de haber sido resultado de una descuidada e inconsciente anticipación del copista de la idea fundamental de la oración ("al çielo"). Sabido es que tal trastrueque involuntario, o error natural, se da muy a menudo en las copias manuscritas antiguas, incluidas las del Libro del Arcipreste ${ }^{41}$.

No tengo solución viable para la hipermetría real del v. 42e ("al que pariste, blanca flor”) como no sea la aplicación al mismo de la llamada ley de Mussafia ${ }^{\star 2}$.

Concluyo con unas reflexiones finales sobre los versos cortos de estos segundos "Gozos de Santa María”, relacionadas con su metría. Hanssen fue el primero que interpretó tales versos como tetrasilábicos en 1902 (art. cit., págs. 207, 228, 232). Desde entonces, los editores que explican la métrica de estas once sextillas simétricas siguen considerando a sus versos de pie quebrado como tetrasilábicos ${ }^{43}$. Con lo que, como consecuencia, para

40 La enmienda que Ag propuso ("fiz", por "fizo") es reprobable por infundada. JR nunca usa el apócope de las formas de $3 .^{\mathrm{a}}$ pers. sing. del perfecto de verbos de radical en $c$-, aunque sí las de $1 .^{\mathrm{a}}$ pers. sing. del dicho perfecto con radical $c$ - (fiz: 19c, 215a, 915a, 947b; dix: 960a, 973c, 1026c, etc.) y las de 3. ${ }^{a}$ pers. sing. del presente de indicativo con tal radical ( $f a z: 70 \mathrm{c}, 156 \mathrm{a}, 157 \mathrm{~b}$, etc.; aduz: 120d), etc.

41 Paralelos trastrueques son, p. ej.: "el que non quiere pagar" $953 \mathrm{~d} S$, frente a "al que pagar non quiere" $G$; "yo le dixe: omillo me" $975 \mathrm{c} G$, en vez de "omíllome, dixe yo" $S$; "a mí non te llegues" $976 \mathrm{~b} G$, en vez de "non te llegues a mí" $S$; "yo le dixe: Por Dios" 982a $G$, frente a "Par Dios, dixe yo" $S$; "yo dixele" 1028a $G$, frente a "yo l' dixe" $S$, etc.

42 Por supuesto, no parece válida la apócope de "pariste" a "parist", puesto que JR no usa jamás tal apócope en las 2 as $^{\text {as }}$ pers. de sing. de perfecto.

43 Véase $L B A C$ ej. pág. XXXV; $L B A C h i$, pág. LI; $L B A C$ Cor, pág. $87 ; L B A \mathrm{Rfl}$, pág. 53; $L B A \mathrm{~W}$ s, pág. XXCIX (entiéndase: LXXXIX); $L B A$ Jaur, pág. 74 ; $\mathrm{MM}$, art. cit., pág. 233 , y $L . B A \mathrm{~J}$, según se infiere por sus vv. $40 \mathrm{c}$ y $40 \mathrm{f}$. 
hacer posible tal medida, se obligan a tener que aplicar el recurso descrito por Mussafia nada menos que a dieciocho versos de los veintidós de pie quebrado que la poesia presente $y$, asimismo, a tener que introducir en $40 \mathrm{f}$ una forzada forma "Spritu" (Hn, Cej, Chi, Cor, Ws y Jt) o "Sprito" (MM), manifiestamente irregular en castellano y, por tanto, a mi juicio, no apta. Difiriendo, pues, de esta opinión general, creo con Ag (Glosario, pág. 98) Que JR compuso estos versos, no con la medida tetrasilábica, sino con la pentasilábica. Ella evita la necesidad de tener que aplicar profusamente un recurso de la lírica provenzal de limitada extensión en la medieval castellana ${ }^{44}$, además de permitir regularizar el verso 40 con la forma apocopada vulgar "Esprito" 4 , explícita una vez en la tradición manuscrita del $L B A$ $(1585 \mathrm{~b} T)$ o, aún mejor, con la forma "Espritu", cuyas primeras letras cons$\tan$ en $11 \mathrm{a} G, 30 \mathrm{c} G, 1640 \mathrm{~d} S$. La medida pentasilábica, por otra parte, requiere en 38c y 40c el deshacer los diptongos "Grabiel" y "Dios" respec-

44 Desde luego, no parece que pueda negarse la aplicación de este recurso a la poesía del $L B A$, en vista de versos como el antedicho $42 \mathrm{e}$, y los $1639 \mathrm{e}, 1639 \mathrm{f}, 1666 \mathrm{~b}$, 1666e, etc. Pero, para un lector actual, es difícil el comprender la aceptación acústicomusical por el poeta medieval de una dualidad de tratamiento métrico, tan dispar, a un mismo tipo de versos, los terminados en voz aguda: unas veces considerados como de ocho sílabas métricas, aunque tengan ocho reales, por estar tratados con arreglo al dicho recurso (42e, 1639f, etc.); pero, en cambio, otras, tenidos como de ocho sílabas métricas y siete reales, por no aplicárseles tal tratamiento $(380 \mathrm{a}, 380 \mathrm{~b}, 851 \mathrm{a}, 851 \mathrm{~d}, 867 \mathrm{~b}$, $1710 \mathrm{~b}, 1710 \mathrm{~d}, 1712 \mathrm{~b}$, etc.).

45 Rfl, Jaur y Blc fijan en sus respectivas ediciones la forma "Spíritu", que, además de la $s$ líquida, a desestimarse, presenta el agravante, en los textos de los dos primeros, de romper la medida tetrasilábica del verso, supuesta por ellos mismos. Blc no deja explícita su idea sobre la métrica de esta poesía. La forma "Espíritu" fijada por Bol es todavía más desviada, porque hace al verso hexasilábico.

16 $\mathrm{Ni}$ que decir tiene que, en los códices del $L B A$, las abreviaciones "Spiritu" $(11 \mathrm{a} S, 30 \mathrm{c} S, 1590 \mathrm{a} T)$ o "Spirit" (1592c $S, 1598 \mathrm{c} S)$ son simples trasvases del uso manuscrito latino corriente en la época y, por tanto, muy comunes (como es natural) en cualquier texto castellano medieval para representar la forma plena hispánica "espíritu" o las vulgares reducidas "espritu"/"esprito". Cej, Chi, Jt y otros editores suelen fijar en sus respectivos textos este sustantivo y su derivado "espiritual" (1591c) con la $s$ líquida, lo que creo un desacierto. Dada la época de la composición del $L B A$, tales voces requieren siempre la expresión de la epentética $e$ - (nótese que el metro de los versos lo permite en todos los casos donde estas voces aparecen), bien bajo la forma espiritu (Pról., rr. 3, 88; vv. 30c, 1585b, 1586b, 1590a, 1596c, 1591d "espiritual", 1636h y 1646f), bien bajo la vulgar espritu/esprito (40f, 1592c y 1598d). Como es sabido, el castellano en su proceso diacrónico, perdió muy tempranamente la fonación independiente de la $s$ líquida (el fenómeno, en realidad, se da ya antes en un amplio sector del latín vulgar de la Romania), precisando de una $e$ - para pronunciarla (estrella $<$ stella, espiga < spica, espina < spina), salvo en algunos cultismos del grupo consonántico inicial sc- en los que la $s$ - se mantiene, oscurecida, durante algún tiempo, por haberse fundido con la $-c$ - inmediata (cetro < sceptrum, centella $<$ scentilla, ciencia $<$ scientia). Luego, no hay razón morfofonética alguna para introducir en el $L B A$ formas con $s$ líquida. Una observación de otro tipo: en 1592cS, Duc, $\mathrm{CrN}$ y Cor leen "sput" (es decir, con signo de abreviación inscrito sobre la $u$ ), lectura, a mi juicio, desacertada. Debe leerse "spirit", al igual que en $1598 \mathrm{c} S$. 
tivamente, a fin de que tales voces se lean como trisílaba la primera ("Grabiel") y como bisilaba la segunda ("Dios") ${ }^{47}$. La trisilabación "Grabiel" nos la asegura, desde luego, la medida de los versos 8c y 38c. Mientras que la forma bisilaba "Dios" se encuentra en los vv. 775b ("Dios vos dé paz, comadre / que por vos vine yo aquí") y 780d ("con lo que l' Dios diere / páselo bien fermoso ${ }^{48}$ : la primera forma, exigida por la metría octosilábica del hemistiquio, y la segunda, por la heptasilábica (aunque ésta, más dudosa, puesto que el hiato pudiera llevarlo "diere" en vez de "Dios"). Por otra parte, la acentuación de Dios sobre la $i$ no debía de ser totalmente extraña a los oídos del castellano medieval, dado que el judío decía Dio y el catalán Déu (en la voz, el corrimiento del acento de primera vocal a segunda está ya consumado en castellano en el siglo xI, si no antes).

El único problema real que plantea la medida pentasilábica de estos pies quebrados es el del v. 40c ("con espanto"), que evidentemente requiere una palabra más de una sola sílaba para ajustarse a tal metro. Esta bien puede ser el adjetivo apocopado "grand", ya sugerido por Ag (Glosario, pág. 119). Voz que nosotros también proponemos como enmienda para el caso, aunque ningún códice del $L B A$ la registra en este lugar. Razones: $10^{\mathbf{a}}$ Nada más fácil en la tradición manuscrita del poema que el perderse por omisión un adjetivo enaltecedor o "engrandecedor" del concepto sustantivo, cuando aquél, en un circunstancial de modo, va calificando a un nombre que, como espanto, suele implicar ya per se una idea de intensa emotividad: "con grand espanto" añade poco, en verdad, a "con espanto". 2. a Los códices dan muestras profusas de la desatención de los escribas en la transcripción de la partícula "grand": en unos puntos del poema este adjetivo se omite; en otros, está alterado de lugar; en otros sustituido por otra voz, y en otros, adicionado indebidamente ${ }^{49}$. En vista de tal mare-magnum de omisiones y errores diversos de tal partícula no parece injustificada la fijación de la indicada enmienda en un verso en el que su metro lo precisa, su contexto lo acepta y la sílaba-voz necesitada en el mismo excluye, creo, cualquier otro término como posible. Al fin y al cabo, tal enmienda (entendidos como pen-

47 El recurso, legítimo en poesía, fue, sin duda, utilizado por JR. Así lo reconocen Chi, Cor y Jt, entre otros editores, por ej., para las siguientes voces: "Grabiel" $8 \mathrm{c}$, $38 \mathrm{c}$; "traïçión" 143a; "sapiençia" 886a, etc.

48 La desdiptongación Dios la fijó en este verso Chi, al que probablemente siguen Ws (quien nunca destaca la eliminación del diptongo con la diéresis correspondiente) y Blc (que siempre fija este signo diacrítico: 8c, 38c, 143a, 199c, 658b, etc.; pero que aquí no se hace, quizá por errata).

49 Véanse algunos ejemplos, sin emitir preferencia personal de variantes: "yaze la falssedat" $69 \mathrm{~b} S$, pero "yaze grant fealdat" $G$; "a las vezes amor" $76 \mathrm{~b} G$, pero "a las vezes grand amor" $S$; "la pascua tiene" $385 \mathrm{~d} S$, pero "grant pascua tien" $G$; "yo ove pereza" $465 \mathrm{a} G$, pero "yo ove grant pereza" $S$; "el uso" $472 \mathrm{~b} S$, pero "grand uso" $G ; 493 \mathrm{~b} G / S ; 597 \mathrm{c} S / G ; 707 \mathrm{c} S / G ; 1094 \mathrm{a} G / S$, etc. 
tasílabos los pies quebrados de esta poesía) es menos disturbadora que la distorsión Spritu/Sprito antedicha más los dieciocho rompimientos de la métrica regular tetrasilábica obligados para quienes enjuician estos versos cortos ruicianos como de cuatro sílabas. Pues lo que, en todo caso, no se puede negar es el que la aplicación de la ley de Mussafia es recurso excepcional de la lírica y quebranto incuestionable de la metría regular del verso.

JosE JuRado

Carleton University. Ottawa, 1985 